Branco MILANOVIC, Inégalités mondiales. Le destin des classes moyennes, les ultra-riches et l'égalité des chances

\title{
Olivier Galland
}

\section{(2) OpenEdition Journals}

Édition électronique

URL : http://journals.openedition.org/ress/5519

DOI : $10.4000 /$ ress.5519

ISBN : 1663-4446

ISSN : $1663-4446$

Éditeur

Librairie Droz

Édition imprimée

Date de publication : 20 décembre 2019

Pagination : 289-291

ISSN : 0048-8046

Référence électronique

Olivier Galland, «Branco MILANOVIC, Inégalités mondiales. Le destin des classes moyennes, les ultrariches et l'égalité des chances ", Revue européenne des sciences sociales [En ligne], 57-2 | 2019, mis en ligne le 20 décembre 2019, consulté le 01 décembre 2020. URL : http://journals.openedition.org/ress/ 5519 ; DOI : https://doi.org/10.4000/ress.5519

Ce document a été généré automatiquement le 1 décembre 2020.

(c) Librairie Droz 


\title{
Branco MILANOVIC, Inégalités mondiales. Le destin des classes moyennes, les ultra-riches et l'égalité des chances
}

\author{
Olivier Galland
}

\section{RÉFÉRENCE}

Branco MILANOVIC, 2019 (2016), Inégalités mondiales. Le destin des classes moyennes, les ultra-riches et l'égalité des chances, Paris, La Découverte, $288 \mathrm{p}$.

Branco Milanovic a été rendu célèbre par sa «courbe de l'éléphant » qui montre l'évolution du revenu réel par fractiles de la distribution des revenus mondiaux entre la chute du mur de Berlin et la crise de 2008. Ce graphique ouvre le livre Global Inequality. A New Approach for the Age of Globalization (Harvard University Press, 2016) qui vient heureusement d'être traduit en français (avec une préface de Thomas Piketty). Cette courbe est d'abord le résultat d'un tour de force méthodologique puisqu'elle résulte de la compilation des enquêtes nationales sur les revenus des ménages de façon à pouvoir traiter la question de l'inégalité mondiale. L'originalité de la démarche de Milanovic (même s'il a été précédé dans cette voie par des chercheurs comme François Bourguignon) se situe là: analyser l'évolution des inégalités entre l'ensemble des habitants de la planète. C'est une tâche éminemment difficile car des données standardisées à ce sujet, qui seraient rassemblées de manière systématique, par exemple par les Nations Unies ou la Banque mondiale, n'existent pas. Milanovic plaide d'ailleurs pour qu'une telle implémentation soit mise en œuvre à l'avenir. Avec son collègue Christoph Lakner, il a donc dû créer de toutes pièces une base de données sur la consommation dans les pays pauvres (les données sur les revenus étant trop parcellaires) et sur les revenus dans les pays riches. 
2 Que montre la courbe de l'éléphant? Tout d'abord, elle a pris ce nom parce que son tracé évoque la forme d'un éléphant qui remonte sa trompe. Plusieurs résultats s'en dégagent. La queue de l'éléphant concerne les plus pauvres des habitants de la planète : leur sort ne s'est pas amélioré relativement au reste de la population mondiale. Un deuxième groupe de perdants est très différent : c'est celui des revenus situés entre le $80^{\mathrm{e}}$ et le $95^{\mathrm{e}}$ percentile (le point le plus bas de la trompe avant qu'elle se relève) - des revenus donc plutôt élevés à l'échelle mondiale (mais pas forcément à l'échelle des pays riches) - qui ont également progressé moins vite que le revenu global; ils se sont relativement appauvris.

3 Il y a enfin les gagnants qui se divisent en deux groupes très différents: les personnes qui se situent autour de la médiane (le dos de l'éléphant) et qui voient leurs revenus réels presque doubler et enfin les $1 \%$ les plus riches de la planète (l'extrémité de la trompe relevée) dont les revenus, déjà très élevés, ont continué de croitre fortement (+65\%) durant la période étudiée. Il faut bien sûr garder à l'esprit qu'on parle ici d'écarts relatifs, ce qui fait que les gains au sommet de la distribution des revenus (le $1 \%)$ sont incomparablement supérieurs à ceux des revenus autour de la médiane. Les gains absolus par tête des $1 \%$ entre les deux dates (en parité de pouvoir d'achat) ont été de 25000 \$, tandis que ceux du revenu médian ont été de 400 \$ ! Les $5 \%$ des plus riches de la planète ont ainsi accaparé $44 \%$ de la croissance du revenu mondial entre les deux dates.

4 Quelle est la composition de ces différents groupes de gagnants et de perdants de la redistribution mondiale des revenus? Les personnes situées autour de la médiane sont, à $90 \%$ issues des pays émergents d'Asie, surtout la Chine, mais aussi l'Indonésie ou la Thaïlande et à un moindre degré l'Inde, une nouvelle classe moyenne mondiale qui émerge. Les perdants quant à eux, et c'est un résultat sans doute moins attendu, sont principalement issus des économies développées et des anciens pays communistes. Mais, même en ne prenant pas en compte ces ex-pays communistes, l'écrasante majorité des membres de ce groupe de « perdants » est issue de «l'ancien monde » des pays riches. Cette classe moyenne des pays développés voit son revenu stagner ou ne plus progresser que faiblement.

5 Pour Milanovic, cette redistribution des revenus à l'échelle mondiale est le résultat conjugué de l'innovation technologique (le progrès technique biaisé disent les économistes pour signifier qu'il profite aux travailleurs qualifiés) et de la mondialisation.

6 La courbe de l'éléphant est évidemment fascinante mais elle a aussi ses limites. Elle a été critiquée sur le plan technique, mais Milanovic et son collègue Lakner ont répondu de façon assez convaincante à ces critiques (voir, par exemple, les critiques de la Resolution Foundation dont le Financial Times a rendu compte < https://www.ft.com/ content/93f2d4ba-7901-11e6-97ae-647294649b28> et la réponse de Lakner: <http:// blogs.worldbank.org/developmenttalk/rebuttal-elephant-graph-discussion>).

Plus important, peut-être, est le fait que, par construction, le monde développé dans son ensemble est concentré dans les derniers déciles de la distribution mondiale des revenus (par exemple, les $5 \%$ les plus pauvres des Américains se situent au $68^{\mathrm{e}}$ percentile), ce qui contribue à rendre presque invisibles les inégalités internes qui s'y jouent (en dehors du $1 \%$ du haut de la distribution). Il est difficile à la fois d'élargir la focale et de la resserrer. Ce que Milanovic appelle donc les «classes moyennes » des pays riches reste un concept assez flou dont on a un peu de mal à cerner les contours. 
On est évidemment tenté de mettre en parallèle les deux évolutions principales que le livre met en lumière - l'émergence de la classe moyenne asiatique et le déclin relatif de la classe moyenne des pays riches - même si l'auteur refuse prudemment d'affirmer un lien de causalité que ses données ne permettent effectivement pas d'établir. La concomitance des deux évolutions n'en est pas moins frappante et est peut-être le signe avant-coureur du basculement de la domination économique de «l'ancien monde » des pays riches vers le « nouveau monde » des pays émergents, essentiellement asiatiques.

Une autre question politique abordée dans l'ouvrage est celle de l'impact du déclin de la classe moyenne occidentale, s'il se confirme, parallèlement à la montée d'une élite mondiale largement détachée de son appartenance nationale, sur la stabilité de la démocratie. Milanovic évoque le risque d'une dérive de la démocratie vers des formes de ploutocratie, d'un gouvernement par les riches. Il ne croit pas du tout que le capitalisme lui-même soit menacé par la montée des inégalités. "La "démondialisation" est impossible, dit-il, parce qu'elle supprimerait la division du travail, l'un des facteurs clés de la croissance économique » (p. 207). Et surtout, aucune alternative crédible n'existe aujourd'hui, argumente-t-il, ce qui fait que «l'hégémonie du capitalisme semble presque impossible à contester». Par contre, l'idée que le capitalisme et la démocratie puissent être « découplés » n'est selon lui pas absurde. Et d'après Milanovic, la montée des inégalités et le déclin des classes moyennes peuvent jouer un rôle dans ce découplage. Selon lui, les classes moyennes sont au cœur du système démocratique en limitant à la fois le pouvoir des riches et des pauvres : " empêcher les riches de leur imposer leurs règles et les pauvres de confisquer leurs biens » (p. 209). Le déclin des classes moyennes conduit à un système qui « ressemble de plus en plus à une ploutocratie" aux États-Unis, et peut aboutir à des formes de populisme en Europe.

10 Mais, un des points sans doute les plus importants qui ressort du travail de Milanovic est la mise en lumière du rôle crucial que joue le pays de naissance dans le destin des individus. Le débat sur l'inégalité se focalise le plus souvent sur les écarts entre groupes sociaux internes à chaque pays, alors que l'enjeu est d'abord, et de plus en plus, mondial et que cette inégalité mondiale est déterminée, avant toute autre caractéristique, par le lieu de naissance des individus. Le travail de Milanovic montre que le lieu de naissance est le plus fort prédicteur du destin social individuel. Il y a donc une très forte rente ou pénalité de citoyenneté selon les termes de l'auteur. Il montre également les implications importantes que ce résultat a sur les migrations. Tout d'abord, c'est évidemment un facteur clé expliquant les mouvements migratoires euxmêmes : si la pénalité de citoyenneté est un facteur décisif qui vous maintient presque à coup sûr dans la pauvreté, fuir son pays de naissance est un comportement simplement rationnel. Il conduit aussi les migrants les moins qualifiés qui se trouveront inévitablement dans le bas de la distribution des revenus du pays d'accueil à choisir les pays riches les plus égalitaires: un effet pervers des États providence les plus développés.

11 Le livre de Milanovic est une contribution capitale au débat sur les inégalités. Il replace utilement ce débat à l'échelle mondiale alors que les travaux sur les inégalités ont trop souvent tendance à ne se focaliser que sur les inégalités internes à chaque entité nationale. Avec la mondialisation et la redistribution des revenus à l'échelle internationale qui l'accompagne, cette lecture purement nationale des inégalités n'est plus adéquate. C'est la grande leçon de cet ouvrage. 


\section{AUTEURS}

OLIVIER GALLAND

Paris, CNRS - GEMASS 